Lancet HIV Commentary

by Intira Jeannie Collins ${ }^{1}$ and Anna Turkova ${ }^{1}$

${ }^{1}$ MRC Clinical Trials Unit at UCL, University College London, United Kingdom

Corresponding author: Intira Jeannie Collins, MRC Clinical Trials Unit at UCL, Institute of Clinical Trials \& Methodology, University College London, 90 High Holborn, London WC1V 6LJNH.

Email: Jeannie.collins@ucl.ac.uk. Tel: +442076704767 


\section{Outcomes in children on raltegravir: a story of two halves}

In the Lancet HIV, Nashman and colleagues present the first long-term safety and efficacy outcomes of children and adolescents on raltegravir in the IMPAACT 1066 trial, a single arm open-label study with over 4 years of follow-up(1). Children with perinatally acquired HIV are recommended to start antiretroviral treatment (ART) from birth and to continue treatment life-long(2). Over time they are inevitably at risk of treatment failure and will require second and subsequent lines of ART(3). Integrase strand transfer inhibitors (INSTI) provide an important treatment option for treatmentexperienced children, including the very young.

Raltegravir is the only INSTI drug option for children younger than 3 years who have previous exposure to non-nucleoside reverse transcriptase inhibitors (NNRTI) and are failing or cannot tolerate protease inhibitor based regimens. Importantly, with rising prevalence of primary NNRTI resistance in newly diagnosed infants in Africa (4), raltegravir is the only non-NNRTI first line treatment option recommended for use from birth. Indeed, the USA paediatric HIV guidelines currently recommend raltegravir as part of the preferred first line regimen from birth to age 6 years(2). WHO also recommends raltegravir as the preferred first line treatment for neonates, and as part of the alternative first and second line ART for older children for whom dolutegravir dosing is not available(5).

However we know raltegravir has important caveats. It requires twice daily dosing in children, it is not available in fixed dose combination with other antiretroviral drugs, it has complex neonatal dosing, and a low genetic resistance barrier which may compromise susceptibility to future INSTI drug options $(6,7)$.

Until now there have been scarce data on long-term safety and efficacy outcomes on raltegravir in ART-experienced children and adolescents. Nashman et al. present outcomes of the IMPAACT 1066 trial which included children across age groups receiving three different formulations: $21 \%$ were aged 4 weeks to $<2$ years who received oral granules; $21 \%$ were aged 2-11 years on chewable tablets; and $52 \%$ were aged $8-18$ years on $400 \mathrm{mg}$ tablets(1). Overall the safety profile was excellent with very low incidence of serious adverse events and toxicity-related discontinuations.

However the virological efficacy results were heterogeneous and the study presents a story of two halves. The good news is that most infants $(n=22)$ who remained on raltegravir did well, with $87 \%$ viral suppression at 240 weeks. This is encouraging and supports the consideration of its use for infants. Although, as Nashman and colleagues noted, this is a single-arm study, and the safety and efficacy of raltegravir in infants has not been directly compared with those starting on other ART regimens. While this study reported good acceptability of RAL among caregivers of infants, it must be noted that RAL has complex dosing for neonates and a complicated preparation process(7). Data on acceptability and feasibility of its use in routine care in resource-limited settings are needed before recommendations for wider use.

The not so good news is the poorer virological outcomes among ART-experienced adolescents, who are a growing population globally (8). Only half of the older children taking raltegravir tablets remained on the drug at 240 weeks, of whom only $44 \%$ were virologically suppressed. One third of all patients with virological failure had resistance to raltegravir which may compromise susceptibility to second generation INSTI. As the authors note, this highlights the need for careful consideration of 
this drug for this challenging age group, taking into account their future drug options. Poor adherence among adolescents is well documented(9), and looking forward, high resistance barrier once-daily fixed dose combination treatments or long-acting injectable drugs will be particularly attractive for this population(10).

As the largest burden of paediatric HIV is centred in sub-Saharan Africa, another key issue to consider is affordability. Raltegravir has limited generic production to date and will need to compete with the low cost dolutegravir-based fixed dose combinations at $\$ 75$ per year(11). There are also questions over how raltegravir in children will fit the goals for alignment of paediatric HIV treatment with WHO adult HIV guidelines, which do not currently include raltegravir as part of the WHO's public health approach(5). Nonetheless, in light of the current limited paediatric treatment options available, particularly in younger age groups, raltegravir-based ART offers an important alternative holding regimen, as we await second generation INSTI to become available across all paediatric age groups. 
1. Nachman S, Alvero C, Teppler H, Homony B, Rodgers AJ, Graham BL, et al. Phase 1/2 open label, non randomised multicentre trial of different Raltegravir formulations in children with HIV-1; Long term safety and efficacy Lancet HIV. 2018;in-press.

2. HIV. PoATaMMoCLw. Guidelines for the Use of Antiretroviral Agents in Pediatric HIV Infection 2018 [updated 22 May 2018. Available from: http://aidsinfo.nih.gov/contentfiles/lvguidelines/pediatricguidelines.pdf.

3. European Pregnancy and Paediatric HIV Cohort Collaboration (EPPICC) study group in EuroCoord. Time to Switch to Second-line Antiretroviral Therapy in Children With Human Immunodeficiency Virus in Europe and Thailand. Clinical infectious diseases : an official publication of the Infectious Diseases Society of America. 2018;66(4):594-603.

4. Jordan MR, Penazzato M, Cournil A, Vubil A, Jani I, Hunt G, et al. Human Immunodeficiency Virus (HIV) Drug Resistance in African Infants and Young Children Newly Diagnosed With HIV: A Multicountry Analysis. Clinical Infectious Diseases. 2017;65(12):2018-25.

5. World Health Organization. Updated recommendations on first-line and seond-line antiretroviral regimens and post-exposure prophylaxis and recommendations on early infant diagnosis of HIV: interim guidance 2018 [Available from:

http://www.who.int/hiv/pub/guidelines/ARV2018update/en/.

6. Fourati $S$, on behalf of the AACRSG, Charpentier C, on behalf of the AACRSG, Amiel C, on behalf of the AACRSG, et al. Cross-resistance to elvitegravir and dolutegravir in 502 patients failing on raltegravir: a French national study of raltegravir-experienced HIV-1-infected patients. Journal of Antimicrobial Chemotherapy. 2015;70(5):1507-12.

7. MERCK. Isentress (raltegravir) for oral suspension: Instructions for use [Available from: https://www.merck.com/product/usa/pi_circulars/i/isentress/isentress_ifu.pdf.

8. The Collaborative Initiative for Paediatric HIV Education and Research (CIPHER) Global Cohort Collaboration. The epidemiology of adolescents living with perinatally acquired HIV: A crossregion global cohort analysis PLoS Med. 2018;15(3).

9. Kim SH, Gerver SM, Fidler S, Ward H. Adherence to antiretroviral therapy in adolescents living with HIV: systematic review and meta-analysis. AIDS. 2014;28(13):1945-56.

10. Penazzato M, Irvine C, Vicari M, Essajee SM, Sharma A, Puthanakit T, et al. A Global Research Agenda for Pediatric HIV. Journal of acquired immune deficiency syndromes (1999). 2018;78 Suppl 1(1):S10-S5.

11. World Health Organization. New high-quality antiretroviral therapy to be launched in South Africa, Kenya and over 90 low- and middle-income countries at reduced price 2017 [updated 21 September 2017. Available from: http://www.who.int/hiv/mediacentre/news/high-quality-arvreduced-price/en/. 